\title{
In Situ Atomic-scale Observation of Thermal-induced Detwinning in Face-centered Cubic Metals
}

Fan Cao, Mingjie Xu, Fan Ye, Thomas Michael Kaufman and Xiaoqing Pan

University of California-Irvine, Irvine, California, United States

Metallic nanomaterials have achieved remarkable applications in various fields, wherein defects, such as twin boundaries, play an enormous role in determining the mechanical [1], optical [2], and catalytic [3] performances. Meanwhile, as film thickness or particle size is reduced, the energy barrier for defect movement usually becomes lower, implying that defects are less stable in nanocrystals, especially at high temperature. However, many metallic nanomaterials work at elevated temperatures, which can facilitate the annealing out of defects and accelerate the material failure. Nevertheless, fundamental understanding of the annealing processes under high temperature still remains elusive due to limited experimental and theoretical techniques. In this context, we investigate the thermal-induced detwinning processes of facecentered cubic (FCC) metals at atomic-scale via in situ aberration-corrected transmission electron microscopy (AC-TEM).

Generally, $\{111\}$ twinning is common and dominant in FCC metals owing to the relatively low stacking fault energy along these close-packed planes. Among various FCC metals, Cu (quasi-noble metal) and Au (noble metal) are two typical species which have attracted remarkable investigations and applications in the past decades. In our experiment, $\mathrm{Cu} / \mathrm{Au}$ nanofilms/nanoparticles were transferred onto Protochips Fusion thermal chips with holey carbon film and the heating process was controlled by the corresponding Protochips software. The in situ atomic-scale observation was conducted on a JEOL-JEM-300CF (doubleaberration-corrected and working at $300 \mathrm{kV}$ ) TEM equipped with a Gatan OneView camera.

Fig. 1a presents a two-layer twin fault in a pristine $\mathrm{Cu}$ nanocrystal at $350{ }^{\circ} \mathrm{C}$, which can be divided into three regions (I, II, and III) by the two parallel twin boundaries (indicated with dashed white lines). Interestingly, the twin fault disappeared abruptly via the displacement of region I and II (Fig. 1b), as depicted with the solid (before transition) and dashed (after transition) white lines, i.e. A slides to $\mathrm{C}$ (or $\mathrm{C}$ to $\mathrm{B}$, or $\mathrm{B}$ to $\mathrm{A}$ ) in region $\mathrm{I}$, and $\mathrm{C}$ to $\mathrm{A}$ in region II. It is likely that region I and II would slide in the closepacked planes $\{111\}$, along $<112>$ directions which are corresponding to the typical partial dislocations with minimized energy in FCC metals (Fig. 1c). Specifically, there might exist two paths for the structural transformation: (1) A1 to $\mathrm{C} 1$ in region I and $\mathrm{C} 1$ to $\mathrm{A} 2$ in region II (solid arrows in Fig. 1c); (2) region I and II move together first (from A1 to B2 and from $\mathrm{C} 1$ to A2, respectively), and then region I continues on from $\mathrm{B} 2$ to $\mathrm{C} 2$ as shown with the dashed arrows in Fig. 1c.

Fig. 2 shows another detwinning process in Au nanocrystals at $400{ }^{\circ} \mathrm{C}$, and similarly, there is a pristine seven-layer twin fault (Fig. 2a). Surprisingly, the twin fault rotates clockwise gradually from the left area (Fig. $2 b$ and c), and then the entire grain changes into a single crystal, except for the generation of a stacking fault (indicted with a black arrowhead in Fig. 2d) due to the twin rotation. Finally, along with the shear moving of the stacking fault layer, a uniform single crystal is generated (Fig. 2e and f). This work revealed new detwinning paths in FCC metal at elevated temperatures, which will facilitate the basic understanding of thermal-induced detwinning mechanisms [4]. 

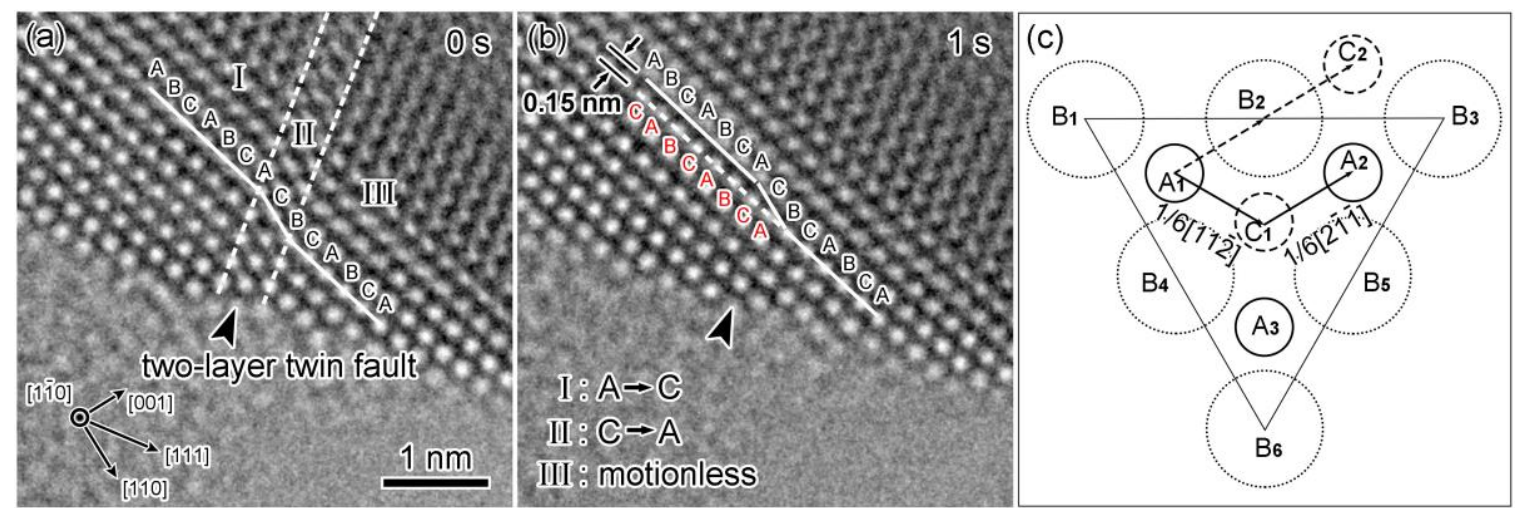

Figure 1. Detwinning process in $\mathrm{Cu}$. (a) Pristine $\mathrm{Cu}$ nanocrystal with a two-layer twin structure. (b) Uniform single-crystal after vanishment of the twin fault. (c) Illustration of the detwinning mechanisms.

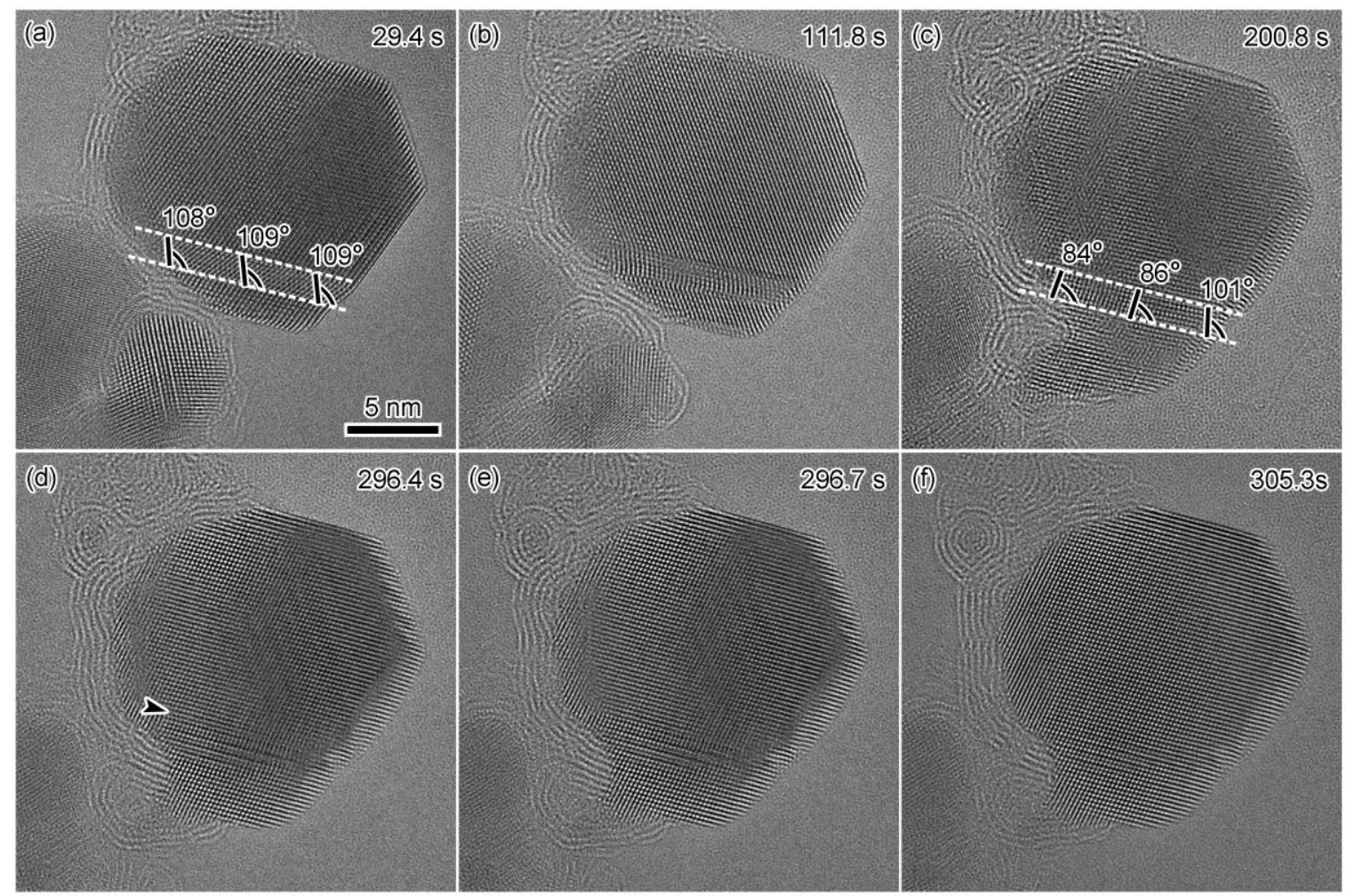

Figure 2. Detwinning process in Au. (a) Pristine Au nanocrystal with a seven-layer twin structure. (b-f) Clockwise rotation of the twin fault, resulting in a uniform single crystal.

\section{References}

[1] K Lu et al., Science 324 (2009), p. 349

[2] A Sánchez-Iglesias et al., J. Am. Chem. Soc. 139 (2017), p. 107.

[3] C Tang et al., ACS Catal. 10 (2020), p. 2026.

[4] This work was supported by Department of Army under Grant W911NF1910263. The in situ TEM experiments were performed at Irvine Materials Research Institute (IMRI), University of California Irvine. The authors acknowledge Horst Hahn for providing the metal samples. 